\title{
A THEOREM ON FACTORIZABLE GROUPS OF ODD ORDER
}

\author{
OSAMU NAGAI
}

To RICHARD BRAUER on his 60 th birthday

Recently, W. Feit [2] obtained some results on factorizable groups of odd order. By using his procedure and applying the theory of R. Brauer [1], we can prove the following theorem similar to that of W. Feit [2]:

TheOREM. Let $G$ be a factorizable group of odd order such that

$$
G=H M
$$

where $H$ is a subgroup of order $3 p, p$ being a prime greater than 3 , and $M$ is a maximal subgroup of $G$. Then $G$ contains a proper normal subgroup which is contained either in $H$ or in $M$.

Proof. It is sufficient to prove the theorem in the case in which $H$ is nonabelian. In fact, if $H$ is abelian, then, as $p \neq 3$, the theorem follows immediately from the theorem of W. Feit [2].

Now, assume that no proper normal subgroup of $G$ is contained in $M$. Suppose that $D=H \cap x M x^{-1} \neq 1$ for some element $x$ in $G$. If $D=H$, then $H \subseteq x M x^{-1}$. Since every subgroup of $G$ conjugate to $M$ is of the form $y M y^{-1}$ for some element $y$ in $H$, it follows that $H$ is contained in every subgroup conjugate to $M$. Hence the intersection of all subgroups conjugate to $M$ is a normal subgroup of $G$, contained in $M$. This contradicts our assumption. Thus $D \neq H$. In this case $H$ is represented as the form $H=A D$, where $A$ is a subgroup of prime order which is either $p$ or 3 . Since the conjugate subgroup $x M x^{-1}$ is the form $y M y^{-1}$ for an element $y$ in $H, G=A \cdot y M y^{-1}$. By a theorem of T. Ikuta [3], either $A$ is normal in $G$ or $y M y^{-1}$ contains a proper normal subgroup of $G$. Thus we can assume that $H \cap x M x^{-1}=1$ for every element $x$ in $G$.

Let $\pi$ be the permutation representation of $G$ induced by the subgroup $M$.

Received January 19, 1962. 
Since the kernel of $\pi$ is contained in $M, \pi$ is faithful. Therefore we can assume that $G$ itself is a transitive permutation group of degree $3 p$. Since $M$ is a maximal subgroup, $G$ is a primitive permutation group. Since $H \cap x M x^{-1}=1$ for every element $x$ in $G, H$ is a regular subgroup of $G$. Since the order of $G$ is odd, $G$ cannot be doubly transitive. Therefore, by the results in [4], $G$ has the following properties:

(a) The order of $G$ contains the prime $p$ to the first power only.

(b) The centralizer of a Sylow $p$-subgroup $P$ is contained in $P$.

(c) $G^{*}$, considered as matrix-representation of $G$, contains no irreducible constituent of degree 1 except the unit representation. Furthermore,

(d) $G^{*}$ contains no irreducible constituents of the exceptional type (in Brauer's sense). In fact, if $G^{*}$ contains an irreducible constituent of exceptional type, then by Theorem 3 of $\mathrm{H}$. Tuan [5], either $G \cong A_{\tau}$ or $G \cong L F(2, p)$. Since the order of $G$ is odd, this is a contradiction.

Under these circumstances, the degrees of the irreducible constituents of $G^{*}$ can be determined completely (see [1], or [4], p. 204). They are $1, p$ and $2 p-1$. Corresponding to this decomposition, the subgroup $G_{1}$ leaving fixed one letter has just three transitive sets whose lengths are $1, v$ and $w$ (see [6], p. 77). Of course $1+v+w=3 p$. If $v=w$, then $3 p=1+2 v$. Since $p-1 \equiv 0$ $(\bmod 3)$, we can put $p-1=6 l$ where $l$ is a rational integer. Then $q=$ $3 p v w / p(2 p-1)=3(9 l+1)^{2} /(12 l+1)$ is not a rational integer. By a theorem of J. S. Frame (see [6], p. 83), this is a contradiction. Hence $v \neq w$.

Now, assume that $1<v<w$. By the methods of $\mathrm{H}$. Wielandt (see [6], in particular p. 92), we obtain the following two equations:

(1) $v+s p+t(2 p-1)=0$,

(2) $v^{2}+s^{2} p+t^{2}(2 p-1)=3 p v$,

where $s$ and $t$ are rational integers. Since $1+v+w=3 p>1+2 v,(3 p-1) / 2>v$. From $(2), t^{2}<3 p v /(2 p-1)<p^{2}$. This means $|t|<p$. From $(1), t \equiv v(\bmod p)$. If we put $t=v+x p$, then, since $v>0$ and $|t|<p, x \leqq 0$. If $x \leqq-3$, then $2 p \leqq p(-x-1)<v$. This is impossible, since $(3 p-1) / 2>v>0$. Hence $x=0$, or -1 , or -2 , that is, $t=v$ or $t=v-p$ or $t=v-2 p$. If $t=v$, then, from (1), $s=-2 t$. Substitute this in (2), $2 t=1$. This is a contradiction. If $t=v-p$, then from (1), s= - 2t-1. From (2) $2 p=6 t^{2}+3 t+1$. On the other hand, 
since $H$ is non-abelian, $p-1 \equiv 0(\bmod 3)$. This is a contradicton. If $t=v-2 p$, then as above, we have $2 p=6 t^{2}+9 t+4$. This is also a contradiction.

Thus the proof is completed.

\section{REFERENCES}

[1] R. Brauer: On permutation groups of prime degree and related classe of groups, Ann. of Math. 44, 57-79 (1943).

[2] W. Feit: A theorem of factorizable groups, Proc. Amer. Math. Soc. 11, 658-659 (1960).

[3] T. Ikuta: Über die Nichteinfachheit einer faktorisierbaren Gruppe, Nat. Sci. Rep. Lib. Arts Fac. Shizucka Univ. 9, 1-2 (1956).

[4] O. Nagai: On transitive groups that contain non-abelian regular subgroups, Osaka Math. J. 13, 199-207 (1961).

[5] H. Tuan: On groups whose orders contain a prime number to the first power, Ann. of Math. 45, 110-140 (1944).

[6] H. Wielandt: Vorlesung über Permutationsgruppen (Ausarbeitung von J. André.) Tübingen 1955.

Department of Mathematics

Yamaguchi University 